\title{
Some Fixed point and common Fixed point Theorems in 2- Banach Spaces
}

\author{
D. P. Shukla ${ }^{1}$ and Vivek Tiwari ${ }^{2}$ \\ Deptt. Of Mathematics/ computer science \\ Govt. P.G. Science college Rewa (M.P.)
}

\begin{abstract}
In this paper we presents some theorems in 2 Banach spaces.
Mathematics subject classification: 47H10, 54H25.

Keywords: Banach Space, 2-Banach Spaces, Fixed point, Common fixed point.
\end{abstract}

\section{Introduction:}

A large variety of the problems of analysis and applied mathematics reduce to finding solutions of non linear functional equations which can be formulated in terms of finding the fixed points of a non linear mapping. Fixed point theorems are very important tools for proving the existence and uniqness of the solutions to various differential, integral and partial differential equations and variational inequalities etc. representing phenomena arising in different fields. Therefore the fixed point methods specially Banach's contraction principle provides a powerful tool for obtaining the solutions of these equations which were very difficult to solve by any other methods. Recently described about the application of Banach's contraction principle [2]. Ghalar [4] introduced the concept of 2- Banach. Recently Badshah and Gupta [3], Yadava, Rajput and Bhardwaj [6] and Yadav, Rajput, Choudhary and Bhardwaj [7] also worked for Banach and 2-Banach spaces for non contraction mapping. In present paper we prove some fixed point theorems for non-contraction mappings, in 2-Banach spaces motivated by above, before starting the main result first we write some definitions

\section{Preliminaries:}

Definition (2.1), 2-Banach Spaces: Let L be a linear 2- normed space to be pair $(L,\|\|,, \|)$ where $\mathrm{L}$ is a linear space and $\|, .$,$\| is non negative, real valued function defined on L$ such that a,b,c, $\in$

$L$.

(i) $\quad\|a, b\|=0$ if and only if a and $\mathrm{b}$ are linearly dependent

(ii) $\quad\|a, b\|=\|b, a\|$

(iii) $\quad\|a, \beta b\|=|\beta|\|a, b\|, \beta$ is real

(iv) $\quad\|a, b+c\| \leq\|a, b\|+\|a, c\|$

Hence $\|.,$.$\| is called a 2$-norm.

Definition (2.2): A sequence $\left\{x_{n}\right\}$ in a linear 2-normed space L, is called a convergent sequence if there is $x \in L$

Such that $\lim _{n \rightarrow \infty}\left\|x_{n}-x, y\right\|=0$ for all $y \in L$.

Definition (2.3): A sequence $\left\{x_{n}\right\}$ in a linear 2-normed space L, is called a Cauchy sequence if there exists $y, z \in L$,

Such that $\mathrm{y}$ and $\mathrm{z}$ are linearly independent and $\lim _{m, n \rightarrow \infty}\left\|x_{m}-x_{n}, y\right\|=0$

Definition (2.4): A linear 2-normed space in which every Cauchy sequence is convergent is called 2-Banach space.

\section{Main Results}

Theorem 3.1: Let $\mathrm{T}$ be a mapping of a 2-Banach spaces into itself. If $\mathrm{T}$ satisfies the following conditions:

$$
F^{2}=I, \quad \text { where } I \text { is } \quad \text { identity }
$$

$$
\begin{array}{r}
\|F x-F y, a\| \leq \alpha\left[\frac{\|x-F x, a\|\|x-y, a\|+\|y-F y, a\|\|y-F x, a\|+\|x-y, a\|^{2}}{\|x-F x, a\|+\|x-y, a\|}\right]+ \\
\beta\left[\frac{\|y-F y, a\|\|x-y, a\|+\|x-F x, a\|\|x-F y, a\|+\|x-y, a\|^{2}}{\|y-F y, a\|+\|x-y, a\|}\right]+
\end{array}
$$


(3.1.2)

$$
\gamma[\|x-F x, a\|+\|y-F y, a\|]+\delta[\|x-F y, a\|+\|y-F x, a\|]+\eta\|x-y, a\|
$$

Where $x \neq y, a>0$ is real with $5 \alpha+5 \beta+4 \gamma+2 \delta+\eta<2$. Then $\mathrm{F}$ has a fixed point. If $\alpha+\beta+2 \delta+$ $\eta<1$.

Then $\mathrm{F}$ has a unique fixed point.

Proof: suppose $\mathrm{x}$ is a point in the 2-Banach space $\mathrm{X}$.

Taking $y=\frac{1}{2}(F+I)(x), Z=F(y)$ and $u=2 y-z$ we have

$\|z-x, a\|=\left\|F y-F^{2}(x), a\right\|=\|F y-F(F x), a\|$

$$
\begin{gathered}
\leq \alpha\left[\frac{\|y-F y, a\|\|y-F x, a\|+\left\|F x-F^{2}(x), a\right\|\|F x-F y, a\|+\|y-F x, a\|^{2}}{\|y-F y\|+\|y-F x\|}\right]+ \\
\beta\left[\frac{\left\|F x-F^{2}(x), a\right\|\|y-F x, a\|+\|y-F y, a\|\left\|y-F^{2}(x), a\right\|+\|y-F x, a\|^{2}}{\left\|F x-F^{2}(x), a\right\|+\|y-F x, a\|}\right] \\
+\gamma\left[\|y-F y, a\|+\left\|F x-F^{2}(x), a\right\|\right]+\delta\left[\left\|y-F^{2}(x), a\right\|+\|F x-F y, a\|\right]+
\end{gathered}
$$

$\eta[\|y-F x, a\|]$

$$
\begin{aligned}
= & \alpha\left[\frac{\|y-F y, a\|\|y-F x, a\|+\|F x-x, a\|\|F x-F y, a\|+\|y-F x, a\|^{2}}{\|y-F y, a\|+\|y-F x, a\|}\right]+ \\
& \beta\left[\frac{\|F x-x, a\|\|y-F x, a\|+\|y-F y, a\|\|y-x, a\|+\|y-F x, a\|^{2}}{\|F x-x, a\|+\|y-F x, a\|}\right]+\gamma\left[\frac{\|y-F y, a\|+\|F x-F(F x), a\|}{2}\right] \\
& +\delta[\|y-x, a\|+\|F x-F y, a\|]+\eta[\|y-F x, a\|] \\
= & \alpha\left[\frac{\|y-F y, a\|\|y-F x, a\|+\|F x-x, a\|\|F x-F y, a\|+\|y-F x, a\|^{2}}{\|F x-F, a\|}\right]+ \\
& \beta\left[\frac{\|F x-x, a\|\|y-F x, a\|+\|y-F y, a\|\|y-x, a\|+\|y-F x, a\|^{2}}{\|y-x, a\|}\right]+\gamma[\|y-F(y), a\|+\|F(X)-X, a\|] \\
& +\delta[\|y-x, a\|+\|F x-F y, a\|]+\eta[\|y-F x, a\|] \\
= & \alpha\left[\frac{\|y-F y, a\|\left\|\frac{1}{2}(F+I)(x)-F x, a\right\|+\|F x-x, a\|\left\|F x-F\left[\frac{1}{2}(F+I)(x), a\right]\right\|+\left\|\frac{1}{2}(F+I)(x)-F x, a\right\|^{2}}{\left\|F x-F\left[\frac{1}{2}(F+I)(x)\right], a\right\|}\right]+ \\
& \beta\left[\frac{\|F x-x, a\|\left\|\frac{1}{2}(F+I)(x)-F x, a\right\|+\|y-F y, a\|\left\|\left[\frac{1}{2}(F+I)(x)-x\right], a\right\|+\left\|\frac{1}{2}(F+I)(x)-F x, a\right\|^{2}}{\left\|\frac{1}{2}(F+I)(x)-x, a\right\|}\right]+ \\
& \gamma[\|y-F y, a\|+\|F x-x, a\|]+\delta\left[\left\|\frac{1}{2}(F+I)(x)-x, a\right\|+\| F x-F\left[\frac{1}{2}(F+\right.\right.
\end{aligned}
$$

$I x, a+$

$$
\begin{aligned}
& \eta\left\|\frac{1}{2}(F+I)(x)-F x, a\right\| \\
= & \alpha\left[\frac{\|y-F y, a\| \frac{1}{2}\|F x-x, a\|+\|F x-x, a\| \frac{1}{2}\|F x-x, a\|+\frac{1}{4}\|F x-x, a\|^{2}}{\frac{1}{2}\|F x-x, a\|}\right]+ \\
& \beta\left[\frac{\|F x-x, a\| \frac{1}{2}\|F x-x, a\|+\|y-F y, a\| \frac{1}{2}\|F x-x, a\|+\frac{1}{4}\|F x-x, a\|^{2}}{\frac{1}{2}\|F x-x, a\|}\right]+ \\
& \gamma[\|y-F y, a\|+\|F x-x, a\|]+\delta\left[\frac{1}{2}\|F x-x, a\|+\frac{1}{2}\|F x-x, a\|\right]+
\end{aligned}
$$

$\frac{\eta}{2}\|F x-x, a\|$

$$
=\alpha\left[\|y-F y, a\|+\|F x-x, a\|+\frac{1}{2}\|F x-x, a\|\right]+
$$

$\beta\left[\|F x-x, a\|+\|y-F y, a\|+\frac{1}{2}\|F x-x, a\|\right]+\gamma[\|y-F y, a\|+\|F x-x, a\|]+\delta\|F x-x, a\|$

$$
\begin{aligned}
+\frac{\eta}{2}\|F x-x, a\| & \\
= & \alpha\left[\|y-F y, a\|+\frac{3}{2}\|F x-x, a\|\right]+\beta\left[\frac{3}{2}\|F x-x, a\|+\|y-F y, a\|\right]+ \\
& \gamma[\|y-F y, a\|+\|F x-x, a\|]+\delta\|F x-x, a\|+\frac{\eta}{2}\|F x-x, a\|
\end{aligned}
$$

$=\left[\frac{3}{2} \alpha+\frac{3}{2} \beta+\gamma+\delta+\frac{\eta}{2}\right]\|F x-x, a\|+[\alpha+\beta+\gamma]\|y-F y, a\|$

$\|z-x, a\| \leq\left[\frac{3}{2} \alpha+\frac{3}{2} \beta+\gamma+\delta+\frac{\eta}{2}\right]\|F x-x, a\|+[\alpha+\beta+\gamma]\|y-F y, a\|$

Also,

$$
\begin{gathered}
\|u-x, a\|=\|2 y-z-x, a\|=\|(F+I)(x)-z-x, a\|=\|F x+x-z-x, a\| \\
=\|F x-z, a\|=\|F x-F y, a\| \\
\|F x-F y, a\| \\
\quad \leq \alpha\left[\frac{\|x-F x, a\|\|x-y, a\|+\|y-F y, a\|\|y-F x, a\|+\|x-y, a\|^{2}}{\|x-F x, a\|+\|x-y, a\|}\right]+
\end{gathered}
$$




$$
\beta\left[\frac{\|y-F(y), a\|\|x-y, a\|+\|x-F(x), a\|\|x-F(y), a\|+\|x-y, a\|^{2}}{\|y-F(y), a\|+\|x-y, a\|}\right]+
$$

$\gamma[\|x-F x, a\|\|y-F y, a\|]+\delta[\|x-F y, a\|+\|y-F x, a\|]+$

$\eta\|x-y, a\|$

$$
\begin{aligned}
\leq & \alpha\left[\frac{\|x-F x, a\|\|x-y, a\|+\|y-F y, a\|\|y-F x, a\|+\|x-y, a\|^{2}}{\|y-F x, a\|}\right]+ \\
& \beta\left[\frac{\|y-F y, a\|\|x-y, a\|+\|x-F x, a\|\|x-F y, a\|+\|x-y, a\|^{2}}{\|x-F y, a\|}\right]+
\end{aligned}
$$

$$
\gamma[\|x-F x, a\|+\|y-F y, a\|]+\delta[\|x-F y, a\|+\|y-F x, a\|]+\quad \eta \| x-
$$

$y, a$

$$
\begin{aligned}
& =\alpha\left[\frac{\|x-F x, a\|\left\|x-\left[\frac{1}{2}(F+I)(x)\right], a\right\|+\|y-F y, a\|\left\|\frac{1}{2}(F+I)(x)-F x, a\right\|+\left\|x-\left[\frac{1}{2}(F+I)(x)\right], a\right\|^{2}}{\left\|\frac{1}{2}(F+I)(x)-F x, a\right\|}\right]+ \\
& \beta\left[\frac{\|y-F y, a\|\left\|x-\left[\frac{1}{2}(F+I)(x)\right], a\right\|+\|x-F x, a\|\left\|x-F\left[\frac{1}{2}(F+I)(x)\right], a\right\|+\left\|x-\left[\frac{1}{2}(F+I)(x)\right], a\right\|^{2}}{\left\|x-F\left[\frac{1}{2}(F+I)(x)\right], a\right\|}\right]+ \\
& \gamma[\|x-F x, a\|+\|y-F y, a\|]+ \\
& \delta\left[\left\|x-F\left[\frac{1}{2}(F+I)(x)\right], a\right\|+\left\|\frac{1}{2}(F+I)(x)-F x, a\right\|\right]+\eta\left[\left\|x-\left[\frac{1}{2}(F+I)(x)\right], a\right\|\right] \\
& =\alpha\left[\frac{\|x-F x, a\| \frac{1}{2}\|x-F x, a\|+\|y-F y, a\| \frac{1}{2}\|x-F x, a\|+\frac{1}{4}\|x-F x, a\|^{2}}{\frac{1}{2}\|x-F x, a\|}\right]+ \\
& \beta\left[\frac{\|y-F y, a\| \frac{1}{2}\|x-F x, a\|+\|x-F x, a\| \frac{1}{2}\|x-F x, a\|+\frac{1}{4}\|x-F x, a\|^{2}}{\frac{1}{2}\|x-F x, a\|}\right]+ \\
& \gamma[\|x-F x, a\|+\|y-F y, a\|]+\delta\left[\frac{1}{2}\|x-F x, a\|+\frac{1}{2}\|x-F x, a\|\right]+ \\
& \eta\left[\frac{1}{2}\|x-F x, a\|\right] \\
& =\alpha\left[\|x-F x, a\|+\|y-F y, a\|+\frac{1}{2}\|x-F x, a\|\right]+ \\
& \beta\left[\|y-F y, a\|+\|x-F x, a\|+\frac{1}{2}\|x-F x, a\|\right]+ \\
& \gamma[\|x-F x, a\|+\|y-F y, a\|]+\delta[\|x-F x, a\|]+\frac{\eta}{2}\|x-F x, a\| \\
& =\alpha\left[\frac{3}{2}\|x-F x, a\|+\|y-F y, a\|\right]+\beta\left[\|y-F y, a\|+\frac{3}{2}\|x-F x, a\|\right] \\
& \gamma[\|x-F x, a\|+\|y-F y, a\|]+\delta[\|x-F x, a\|]+\frac{\eta}{2}\|x-F x, a\| \\
& =\left[\frac{3}{2} \alpha+\frac{3}{2} \beta+\gamma+\delta+\frac{\eta}{2}\right]\|x-F x, a\|+[\alpha+\beta+\gamma]\|y-F y, a\|
\end{aligned}
$$

$\therefore\|u-x, a\| \leq\left[\frac{3}{2} \alpha+\frac{3}{2} \beta+\gamma+\delta+\frac{\eta}{2}\right]\|x-F x, a\|+[\alpha+\beta+\gamma]\|y-F y, a\|$

Now,

$\|z-u, a\| \leq\|z-x, a\|+\|x-u, a\|$

$$
\begin{aligned}
= & {\left[\frac{3}{2} \alpha+\frac{3}{2} \beta+\gamma+\delta+\frac{\eta}{2}\right]\|x-F x, a\|+[\alpha+\beta+\gamma]\|y-F y, a\|+} \\
& {\left[\left[\frac{3}{2} \alpha+\frac{3}{2} \beta+\gamma+\delta+\frac{\eta}{2}\right]\|x-F x, a\|+[\alpha+\beta+\gamma]\|y-F y, a\|\right] } \\
= & 2\left[\frac{3}{2} \alpha+\frac{3}{2} \beta+\gamma+\delta+\frac{\eta}{2}\right]\|x-F x, a\|+2[\alpha+\beta+\gamma]\|y-F y, a\| \\
= & {[3 \alpha+3 \beta+2 \gamma+2 \delta+\eta]\|x-F x, a\|+[2 \alpha+2 \beta+2 \gamma]\|y-F y, a\| }
\end{aligned}
$$

$\|z-u, a\|=[3 \alpha+3 \beta+2 \gamma+2 \delta+\eta]\|x-F x, a\|+[2 \alpha+2 \beta+2 \gamma]\|y-F y, a\|$

Also, $\|z-u, a\|=\|F y-(2 y-z), a\|$

$$
\begin{aligned}
& =\|F y-2 y+z, a\| \\
& =2\|F y-y, a\|
\end{aligned}
$$

$\therefore$ From (3.1.5)

$\therefore 2\|y-F y, a\|=[3 \alpha+3 \beta+2 \gamma+2 \delta+\eta]\|x-F x, a\|+[2 \alpha+2 \beta+2 \gamma]\|y-F y, a\|$

$\therefore\|y-F y, a\| \leq q\|x-F x, a\|$

Where $\mathrm{q}=\frac{3 \alpha+3 \beta+2 \gamma+2 \delta+\eta}{2-(2 \alpha+2 \beta+2 \gamma)}<1$

Since $5 \alpha+5 \beta+4 \gamma+2 \delta+\eta<2$

Let $\mathrm{G}=\frac{1}{2}(F+I)$ then for every $x \in X$

$\left\|G^{2}(x)-G(x), a\right\|=\|G(y)-y, a\|$

$$
=\left\|\frac{1}{2}(F+I)(y)-y, a\right\|
$$




$$
\begin{aligned}
& =\frac{1}{2}\|y-F y, a\| \\
& <\frac{q}{2}\|x-F x, a\|
\end{aligned}
$$

By the definition of $\mathrm{q}$, we claim that $\left\{G^{2}(x)\right\}$ is a Cauchy sequence in $\mathrm{X}$.

By the completeness, $\left\{G^{2}(x)\right\}$ converges to some element $x_{0}$ in $\mathrm{X}$.

Which implies that $G\left(x_{0}\right)=x_{0}$.

$$
\text { i.e. } \lim _{n \rightarrow \infty} G^{n}(x)=x_{0}
$$

Hence $F\left(x_{0}\right)=x_{0}$

i.e $x_{0}$ is a fixed point of $\mathrm{F}$.

For the uniqueness, if possible let $y_{0}\left(\neq x_{0}\right)$ be another fixed point of $F$ then

$\left\|x_{0}-y_{0}, a\right\|=\left\|F\left(x_{0}\right)-F\left(y_{0}\right), a\right\|$

$$
\begin{aligned}
\leq & \alpha\left[\frac{\left\|x_{0}-F\left(x_{0}\right), a\right\|\left\|x_{0}-y_{0}, a\right\|+\left\|y_{0}-F\left(y_{0}\right), a\right\|\left\|y_{0}-F\left(x_{0}\right), a\right\|+\left\|x_{0}-y_{0}, a\right\|^{2}}{\left\|x_{0}-F\left(x_{0}\right), a\right\|+\left\|x_{0}-y_{0}, a\right\|}\right]+ \\
& \beta\left[\frac{\left\|y_{0}-F\left(y_{0}\right), a\right\|\left\|x_{0}-y_{0}, a\right\|+\left\|x_{0}-F\left(x_{0}\right), a\right\|\left\|x_{0}-F\left(y_{0}\right), a\right\|+\left\|x_{0}-y_{0}, a\right\|^{2}}{\left\|y_{0}-F\left(y_{0}\right), a\right\|+\left\|x_{0}-y_{0}, a\right\|}\right] \\
& \gamma\left[\left\|x_{0}-F\left(x_{0}\right), a\right\|+\left\|y_{0}-F\left(y_{0}\right), a\right\|\right]+ \\
& \delta\left[\left\|x_{0}-F\left(y_{0}\right), a\right\|+\left\|y_{0}-F\left(y_{0}\right), a\right\|\right]+\eta\left\|x_{0}-y_{0}, a\right\| \\
= & \alpha \frac{\left\|x_{0}-y_{0}, a\right\|^{2}}{\left\|x_{0}-y_{0}, a\right\|}+\beta \frac{\left\|x_{0}-y_{0}, a\right\|^{2}}{\left\|x_{0}-y_{0}, a\right\|}+2 \delta\left\|x_{0}-y_{0}\right\|+\eta\left\|x_{0}-y_{0}\right\| \\
= & \alpha\left\|x_{0}-y_{0}, a\right\|+\beta\left\|x_{0}-y_{0}, a\right\|+2 \delta\left\|x_{0}-y_{0}, a\right\|+\eta\left\|x_{0}-y_{0}, a\right\| \\
= & {[\alpha+\beta+2 \delta+\eta]\left\|x_{0}-y_{0}, a\right\| }
\end{aligned}
$$

$\therefore\left\|x_{0}-y_{0}, a\right\| \leq[\alpha+\beta+2 \delta+\eta]\left\|x_{0}-y_{0}, a\right\|$

Since $\alpha+\beta+2 \delta+\eta<1$

$\therefore\left\|x_{0}-y_{0}, a\right\|=0$

$\therefore x_{0}=y_{0}$

This is the complete prof.

Theorem 3.2 : Let $\mathrm{K}$ be closed and convex subject of a Banach space X. Let $F: K \rightarrow K, G: K \rightarrow K$

Satisfy the following conditions:

$\mathrm{F}$ and $\mathrm{G}$ commute

$F^{2}=$ and $G^{2}=I$, where I denotes identify mapping

$\|F(x)-F(y), a\|$

$\leq \alpha\left[\frac{\|G(x)-F(x), a\|\|G(x)-G(y), a\|+\|G(y)-F(y), a\|\|G(y)-F(x), a\|+\|G(x)-G(y), a\|^{2}}{\|G(x)-F(x), a\|+\|G(x)-G(y), a\|}\right]$

$+\beta\left[\frac{\|G(y)-F(y), a\|\|G(x)-G(y), a\|+\|G(x)-F(x), a\|\|G(x)-F(y), a\|+\|G(x)-G(y), a\|^{2}}{\|G(y)-F(y), a\|+\|G(x)-G(y), a\|}\right]$

$+\gamma[\|G(x)-F(x), a\|+\|G(y)-F(y), a\|]+\delta[\|G(x)-F(x), a\|+\|G(y)-F(x), a\|]$

$+\eta\|G(x)-G(y), a\|$

For every $x, y \in X, a>0$ is real and $5 \alpha+5 \beta+4 \gamma+2 \delta+\eta<2$. Then there exist at least one fixed point, $x_{0} \in X$ such that $\mathrm{F}\left(x_{0}\right)=G\left(x_{0}\right)=x_{0}$. Further if $\alpha+\beta+2 \delta+\eta<1$ then $\mathrm{X}$ is the unique fixed point of $\mathrm{F}$ and $\mathrm{G}$.

Proof: From (3.2.1) and (3.2.2) it follows that $(F G)^{2}=I$ and (3.2.2) and (3.2.3) weget

$\|F G G(x)-F G G(y), a\|=\left\|F G^{2}(x)-F G^{2}(y), a\right\|$

$\leq \alpha\left[\begin{array}{c}\left\|G G^{2}(x)-F G^{2}(x), a\right\|\left\|G G^{2}(x)-G G^{2}(y), a\right\|+\left\|G G^{2}(y)-F G^{2}(y), a\right\|\left\|G G^{2}(y)-F G^{2}(x), a\right\| \\ +\left\|G G^{2}(x)-G G^{2}(y), a\right\|^{2}\end{array}\right]$

$+\beta\left[\frac{\begin{array}{c}\left\|G G^{2}(y)-F G^{2}(y), a\right\|\left\|G G^{2}(x)-G G^{2}(y), a\right\|+\left\|G G^{2}(x)-F G^{2}(x), a\right\|\left\|G G^{2}(x)-F G^{2}(y), a\right\| \\ +\left\|G G^{2}(x)-G G^{2}(y), a\right\|^{2}\end{array}}{\left\|G G^{2}(y)-F G^{2}(y), a\right\|+\left\|G G^{2}(x)-G G^{2}(y), a\right\|}\right]$

$+\gamma\left[\left\|G G^{2}(x)-F G^{2}(x), a\right\|+\left\|G G^{2}(y)-F G^{2}(y), a\right\|\right]$

$+\delta\left[\left\|G G^{2}(x)-F G^{2}(y), a\right\|+\left\|G G^{2}(y)-F G^{2}(x), a\right\|\right]+\eta\left\|G G^{2}(x)-G G^{2}(y), a\right\|$ 


$$
\begin{aligned}
& \leq \alpha\left[\begin{array}{c}
\|G(x)-F G \cdot G(x), a\|\|G(x)-G(y), a\|+\|G(y)-F G \cdot G(y), a\|\|G(y)-F G \cdot G(x), a\|+ \\
\|G(x)-G(y), a\|^{2} \\
+\beta G(x)-F G \cdot G(x), a\|+\| G(x)-G(y), a \|
\end{array}\right] \\
& {\left[\begin{array}{c}
\|G(y)-F G \cdot G(y), a\|\|G(x)-G(y), a\|+\|G(x)-F G \cdot G(x), a\|\|G(x)-F G \cdot G(y), a\|+ \\
\|G(x)-G(y), a\|^{2}
\end{array}\right]}
\end{aligned}
$$

$+\gamma[\|G(x)-F G \cdot G(x), a\|+\|G(y)-F G \cdot G(y), a\|]$

$+\delta[\|G(x)-F G \cdot G(y), a\|+\|G(y)-F G \cdot G(x), a\|]+\eta[\|G(x)-G(y), a\|]$

Now that $G(x)=Z$ and $G(y)=W$, then we get

$\|F G(z)-F G(w), a\|$

$$
\leq \alpha\left[\frac{\|z-F G(z), a\|\|z-w, a\|+\|w-F G(w), a\|\|w-F G(z), a\|+\|z-w, a\|^{2}}{\|z-F G(z), a\|+\|z-w, a\|}\right]+
$$

$\beta\left[\frac{\|w-F G(w), a\|\|z-w, a\|+\|z-F G(z), a\|\|z-F G(w), a\|+\|z-w, a\|^{2}}{\|w-F G(w), a\|+\|z-w, a\|}\right]+$

$\gamma[\|z-F G(z), a\|+\|w-F G(w), a\|]+\delta[\|z-F G(w), a\|+\|w-F G(z), a\|]+$

$\eta\|z-w, a\|$

We have $(F G)^{2}=I$ and so by theorem 1 . FG has at least one fixed point say $x_{0}$ in K, i.e.

$$
F G\left(x_{0}\right)=x_{0}
$$

$F F G\left(x_{0}\right)=F\left(x_{0}\right)$

$\mathrm{G}\left(x_{0}\right)=F\left(x_{0}\right)$

Now,

$\left\|F\left(x_{0}\right)-x_{0}, a\right\|=\left\|F\left(x_{0}\right)-F^{2}\left(x_{0}\right), a\right\|=\left\|F\left(x_{0}\right)-F F\left(x_{0}\right), a\right\|$

$\leq \alpha\left[\begin{array}{c}\left\|G\left(x_{0}\right)-F\left(x_{0}\right), a\right\|\left\|G\left(x_{0}\right)-G F\left(x_{0}\right), a\right\|+\left\|G F\left(x_{0}\right)-F F\left(x_{0}\right), a\right\|\left\|G F\left(x_{0}\right)-F\left(x_{0}\right), a\right\| \\ +\left\|G\left(x_{0}\right)-G F\left(x_{0}\right), a\right\|^{2}\end{array}\right]$
$+\beta\left[\begin{array}{c}\left\|G\left(x_{0}\right)-F\left(x_{0}\right), a\right\|+\left\|G\left(x_{0}\right)-G F\left(x_{0}\right), a\right\| \\ \frac{\left\|G\left(x_{0}\right)-F F\left(x_{0}\right), a\right\|\left\|G\left(x_{0}\right)-G F\left(x_{0}\right), a\right\|+\left\|G\left(x_{0}\right)-F\left(x_{0}\right), a\right\|\left\|G\left(x_{0}\right)-F F\left(x_{0}\right), a\right\|}{+\left\|G\left(x_{0}\right)-G F\left(x_{0}\right), a\right\|^{2}}\end{array}\right]$

$+\gamma\left[\left\|G\left(x_{0}\right)-F\left(x_{0}\right), a\right\|+\left\|G F\left(x_{0}\right)-F F\left(x_{0}\right), a\right\|\right]$

$+\delta\left[\left\|G\left(x_{0}\right)-F F\left(x_{0}\right), a\right\|+\left\|G F\left(x_{0}\right)-F\left(x_{0}\right), a\right\|\right]+\eta\left\|G\left(x_{0}\right)-G F\left(x_{0}\right), a\right\|$

$=\alpha\left[\frac{\left\|F\left(x_{0}\right)-F\left(x_{0}\right), a\right\|\left\|F\left(x_{0}\right)-x_{0}, a\right\|+\left\|x_{0}-x_{0}, a\right\|\left\|x_{0}-F\left(x_{0}\right), a\right\|+\left\|F\left(x_{0}\right)-x_{0}, a\right\|^{2}}{\left\|F\left(x_{0}\right)-F\left(x_{0}\right), a\right\|+\left\|F\left(x_{0}\right)-x_{0}, a\right\|}\right]$

$+\beta\left[\frac{\left\|x_{0}-x_{0}, a\right\|\left\|F\left(x_{0}\right)-x_{0}, a\right\|+\left\|F\left(x_{0}\right)-F\left(x_{0}\right), a\right\|\left\|F\left(x_{0}\right)-x_{0}, a\right\|+\left\|F\left(x_{0}\right)-x_{0}, a\right\|^{2}}{\left\|x_{0}-x_{0}, a\right\|+\left\|F\left(x_{0}\right)-x_{0}, a\right\|}\right]$

$+\gamma\left[\left\|F\left(x_{0}\right)-F\left(x_{0}\right), a\right\|+\left\|x_{0}-x_{0}, a\right\|\right]+\delta\left[\left\|F\left(x_{0}\right)-x_{0}, a\right\|+\left\|x_{0}-F\left(x_{0}\right), a\right\|\right]$

$+\eta\left[\left\|F\left(x_{0}\right)-x_{0}, a\right\|\right]$

$=\alpha\left\|F\left(x_{0}\right)-x_{0}, a\right\|+\beta\left\|F\left(x_{0}\right)-x_{0}, a\right\|+2 \delta\left\|F\left(x_{0}\right)-x_{0}, a\right\|+\eta\left\|F\left(x_{0}\right)-x_{0}, a\right\|$

$=(\alpha+\beta+2 \delta+\eta)\left\|F\left(x_{0}\right)-x_{0}, a\right\|$

Therefore

$\left\|F\left(x_{0}\right)-x_{0}, a\right\| \leq(\alpha+\beta+2 \delta+\eta)\left\|F\left(x_{0}\right)-x_{0}, a\right\|$

This is contradiction

Since $\alpha+\beta+\delta+\eta<1$

$\therefore F\left(x_{0}\right)=x_{0}$

i.e. $x_{0}$ is fixed point of $\mathrm{F}$ but $F\left(x_{0}\right)=G\left(x_{0}\right)$ therefore we have $G\left(x_{0}\right)=x_{0}$

i.e. $x_{0}$ is the common fixed point of $\mathrm{F}$ and $\mathrm{G}$.

Now we shall prove that $x_{0}$ is the unique common fixed point of $\mathrm{F}$ and $\mathrm{G}$. If possible let $y_{0}$ be another fixed point of $\mathrm{F}$ and $\mathrm{G}$.

Now by (3.2.1), (3.2.2), (3.2.3), (3.2.4) and (3.2.5) we have

$\left\|x_{0}-y_{0}, a\right\|=\left\|F^{2}\left(x_{0}\right)-F^{2}\left(y_{0}\right), a\right\|=\left\|F F\left(x_{0}\right)-F F\left(y_{0}\right), a\right\|$ 


$\leq \alpha\left[\begin{array}{c}\left\|G F\left(x_{0}\right)-F F\left(x_{0}\right), a\right\|\left\|G F\left(x_{0}\right)-G F\left(y_{0}\right), a\right\|+\left\|G F\left(y_{0}\right)-F F\left(y_{0}\right), a\right\|\left\|G F\left(y_{0}\right)-F F\left(x_{0}\right), a\right\| \\ +\left\|G F\left(x_{0}\right)-G F\left(y_{0}\right), a\right\|^{2}\end{array}\right]$
$+\beta\left[\begin{array}{c}\left\|G\left(x_{0}\right)-F\left(x_{0}\right), a\right\|+\left\|G\left(x_{0}\right)-G F\left(x_{0}\right), a\right\| \\ \left\|G F\left(y_{0}\right)-F F\left(y_{0}\right), a\right\|\left\|G F\left(x_{0}\right)-G F\left(y_{0}\right), a\right\|+\left\|G F\left(x_{0}\right)-F F\left(x_{0}\right), a\right\|\left\|G F\left(x_{0}\right)-F F\left(y_{0}\right), a\right\| \\ +\left\|G F\left(x_{0}\right)-G F\left(y_{0}\right), a\right\|^{2}\end{array}\right]$

$+\gamma\left[\left\|G F\left(x_{0}\right)-F F\left(x_{0}\right), a\right\|+\left\|G F\left(y_{0}\right)-F F\left(y_{0}\right), a\right\|\right]$

$+\delta\left[\left\|G F\left(x_{0}\right)-F F\left(y_{0}\right), a\right\|+\left\|G F\left(y_{0}\right)-F F\left(x_{0}\right), a\right\|\right]+\eta\left\|G F\left(x_{0}\right)-G F\left(y_{0}\right), a\right\|$

$=\alpha\left\|x_{0}-y_{0}, a\right\|+\beta\left\|x_{0}-y_{0}, a\right\|+2 \delta\left\|x_{0}-y_{0}, a\right\|+\eta\left\|x_{0}-y_{0}, a\right\|$

$=(\alpha+\beta+2 \delta+\eta)\left\|x_{0}-y_{0}, a\right\|$

$\left\|x_{0}-y_{0}, a\right\| \leq(\alpha+\beta+2 \delta+\eta)\left\|x_{0}-y_{0}, a\right\|$

Since $\alpha+\beta+2 \delta+\eta<1$. it follows,

$x_{0}=y_{0}$.

Proving the uniqueness of $x_{0}$.

Hence the complete proof of theorem 2.

\section{References}

[1]. Ahmad, and Shakil, M. "Some fixed point theorems in Banach spaces" Nonlinear Funct.Anal. \& Appl. 11 (2006) 343-349.

[2]. Banach, S. "Surles operation dans les ensembles abstraits et leur application aux equations integrals" Fund. Math.3 (1922) 133-181.

[3]. Badshah, V.H. and Gupta, O.P. "Fixed point theorems in Banach and 2-Banach spaces" Jnanabha 35 (2005) 73-78.

[4]. Gahlar, S. "2-metrche raume and ihre topologiscche structure" Math.Nadh.26 (1963-64) 115-148.

[5]. Verma, B.P. “Application of Banach fixed point theorem to solve non linear equations and its generalization" Jnanabha 36 (2006) 21-23.

[6]. Yadava, R.N., Rajput, S.S. and Bhardwaj, R.K. "Some fixed point and common fixed point theorem in Banach spaces" Acta Ciencia Indica 33 No 2 (2007) 453-460.

[7]. Yadava, R.N., Rajput, S.S.,Choudhary, S. and Bhardwaj, R.K. "Some fixed point and common fixed point theorems for noncontraction mapping on 2-Banach spaces" Acta Ciencia Indica 33 No 3 (2007) 737-744. 